Vol. 14, $\mathrm{N}^{\circ} 2$, pp. 105-113, December 1995

Universidad Católica del Norte

Antofagasta - Chile

\title{
CREATION OF SOME STRUCTURES IN CATEGORIES
}

\author{
H. A. S. Abujabal, M. A. Obaid \\ King Abdul Aziz University, Saudi Arabia \\ and \\ S. M. A. Zaidi \\ Aligrah Muslim University, India
}

\begin{abstract}
In this paper, we study the creation property of projective and forgetful functors via generalized pullback $(G P B)$ and generalized pushout (GPO) structures and also discus some general results.
\end{abstract}

*Principle author 
In Maclane [3] and Schubert [4] the concept of creation of limits via diagram functors has been discussed. Here, we study the creation of generalized pullback (GPB) and generalized pushout (GPO) structures through cones. We have proved that the projective functors from comma categories, morph categories and forgetful functors create GPB as well as GPO. In particular, we have shown if a functor creates GPB and the range category is also GPB category, then the functor and the domain category are both GPB structures.

Definition 1. A non-empty family of morphisms $\left\{\alpha_{i}: A \rightarrow A_{i}\right\}_{I}$ in a category $\mathcal{C}$ is called a left cone over $I$ with vertex $A$ and base objects $A_{i}, i \in I$. We will denote it by $\left[A, \alpha_{i}, A_{i}\right]_{I}$. Dually, a non-empty family of morphisms $\left\{\alpha_{i}: A_{i} \rightarrow A\right\}_{I}$ in a category $\mathcal{C}$ is called a right cone over $I$ with vertex $A$ and base objects $A_{i}, i \in I$. We will denote it by $\left[A_{i}, \alpha_{i}, A\right]_{I}$.

Definition 2. In a category $\mathcal{C}$, a left cone $\left[P, \alpha_{i}, B_{i}\right]_{I}$ is called the generalized pullback (GPB) of the right cone $\left[B_{i}, \beta_{i}, A\right]_{I}$ if and only if GPB $_{1}$ : $\beta_{j \alpha j}=\beta_{k} \alpha_{k}$ for all $j, k \in I$,

$\mathrm{GPB}_{2}:$ For any other left cone $\left[X, f_{i}, B_{i}\right]_{I}$ in $\mathcal{C}$ with $\beta_{j} f_{j}=\beta_{k} f_{k}$ for all $j, k \in I$, there exists a unique morphism $\eta: X \rightarrow P$ in $\mathcal{C}$ such that $\alpha_{i} \eta=f_{i}$ for all $i \in I$.

Notation. GPB $\left[B_{i}, \beta_{i}, A\right]_{I}=\left[P, \alpha_{i}, \beta_{i}\right]_{I}$.

Dually, we can define generalized pushout (GPO).

Definition 3. A category $\mathcal{C}$ will be called a GPB category if for every right cone of morphisms in $\mathcal{C}$, there is a GPB in $\mathcal{C}$.

Dually we define GPO category.

Definition 4. A covariant functor $F: \mathcal{C} \rightarrow \mathcal{D}$ will be called a GPB functor if it preserves the GPB structure, that is if $\operatorname{GPB}\left[B_{i}, \beta_{i}, A\right]_{I}=\left[P, \alpha_{i}, B_{i}\right]_{I}$ in $\mathcal{C}$, then

$$
\mathrm{GPB}\left[F\left(B_{i}\right), F\left(\beta_{i}\right), F(A)\right]_{I}=\left[F(P), F\left(\alpha_{i}\right), F\left(B_{i}\right)\right]_{I}
$$

in $\mathcal{D}$.

Dually, GPO functor can be defined. 
Definition 5. A functor $F: \mathcal{A} \rightarrow \mathcal{C}$ creates GPB for a right cone $\left[A_{i}, \alpha_{i}, A\right]_{I}$ in $\mathcal{A}$, if

(i) For every GPB $\left[F\left(A_{i}\right), F\left(\alpha_{i}\right), F(A)\right]_{I}=\left[P^{\prime}, \beta_{i}^{\prime}, F\left(A_{i}\right)\right]_{I}$ in $\mathcal{C}$, there is exactly one left cone $\left[P, \beta_{i}, A_{i}\right]_{I}$ in $\mathcal{A}$ such that $F(P)=P^{\prime}$, and $F\left(\beta_{i}\right)=\beta_{i}^{\prime}$ for all $i \in I$.

(ii) The left cone $\left[P, \beta_{i}, A_{i}\right]_{I}$ is a GPB of the right cone $\left[A_{i}, \alpha_{i}, A\right]_{I}$ in $\mathcal{A}$, that is

$$
\mathrm{GPB}\left[A_{i}, \alpha_{i}, A\right]_{I}=\left[P, \beta_{i}, A_{i}\right]_{I} \text { in } \mathcal{A} \text {. }
$$

Dually,

Definition 5*. A functor $F: \mathcal{A} \rightarrow \mathcal{C}$ creates GPO for a left cone $\left[C, \gamma_{i}, C_{i}\right]_{I}$ in $\mathcal{A}$, if

(i) For every $\operatorname{GPO}\left[F(C), F\left(\gamma_{i}\right), F\left(C_{i}\right)\right]_{I}=\left[F\left(C_{i}\right), \delta_{i}^{\prime}, Q^{\prime}\right]_{I}$ in $\mathcal{C}$, there is exactly one right cone $\left[C_{i}, \delta_{i}, Q\right]_{I}$ in $\mathcal{A}$, such that $F(Q)=Q^{\prime}$ and $F\left(\delta_{i}\right)=\delta_{i}^{\prime}$ for all $i \in I$.

(ii) The right cone $\left[C_{i}, i, Q\right]_{I}$ is a GPO of the left cone $\left[C, \gamma_{i}, C_{i}\right]_{I}$ in $\mathcal{A}$, that is

$$
\operatorname{GPO}\left[C, \gamma_{i}, C_{i}\right]_{I}=\left[C_{i}, i, Q\right] \text { in } \mathcal{A} \text {. }
$$

Proposition 1. For any category $\mathcal{C}$ the projection functor $(X \downarrow \mathcal{C}) \rightarrow \mathcal{C}$ of the comma category creates GPB.

Proof. The proof follows directly from the fact that this functor is tripleable [4, Theorem 21. 3.9], and indeed this functor creates all limits.

Indeed, we consider a right cone $\left[\left(f_{i}, A_{i}\right), \alpha_{i},(f, A)\right]_{I}$ in the comma category $(X \downarrow \mathcal{C})$. Then the projection of this cone is a right cone $\left[A_{i}, \alpha_{i}, A\right]_{I}$ in $C$. Suppose $\operatorname{GPB}\left[A_{i}, \alpha_{i}, A\right]_{I}=\left[P, \beta_{i}, A_{i}\right]_{I}$ in $C$. By the definition of morphism in comma category, for every $i \in I$, we have a commutative diagram

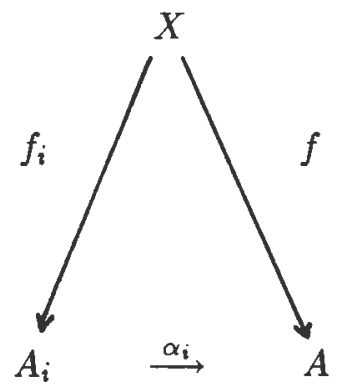




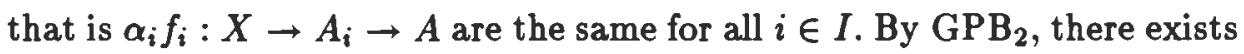
a unique morphism $\eta: X \rightarrow P$ such that $\beta_{i} \eta=f_{i}$ for all $i \in I$. Thus we get a left cone $\left[(\eta, P), \beta_{i},\left(f_{i}, A_{i}\right)\right]_{I}$ in the comma category $(X \downarrow \mathcal{C})$ whose projection in the category $\mathcal{C}$ is the left cone $\left[P, \beta_{i}, A_{i}\right]_{I}$ and $\alpha_{i} \beta_{i}:(\eta, P) \rightarrow$ $\left(f_{i}, A_{i}\right) \rightarrow(f, A)$ are the same for all $i \in I$. Suppose there is a left cone $\left[(g, Y), \gamma_{i},\left(f_{i}, A_{i}\right)\right]_{I}$ in $(X \downarrow \mathcal{C})$ such that the morphisms $\alpha_{i} \gamma_{i}:(g, Y) \rightarrow$ $\left(f_{i}, A_{i}\right) \rightarrow(f, A)$ are the same for all $i \in I$. By applying the projection functor, we get that the morphisms $\alpha_{i} \gamma_{i}: Y \rightarrow A_{i} \rightarrow A$ are the same for all $i \in I$. By $\mathrm{GPB}_{2}$, there exists a unique morphism $\xi: Y \rightarrow P$ such that $\beta_{i} \xi=\gamma_{i}: Y \rightarrow A_{i}$ for all $i \in I$. For each $i \in I$, we have a diagram

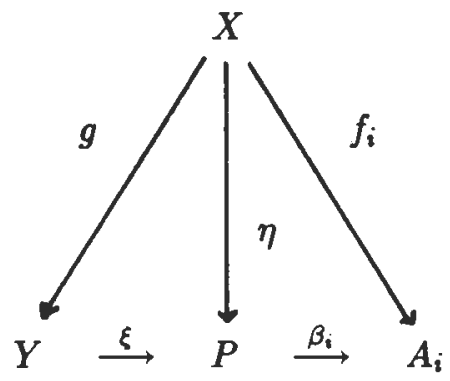

in which outer triangle and the triangle in right hand side are commutative, that is $\beta_{i} \eta=\beta_{i}(\xi g)=f_{i}$ for all $i \in I$. But the factorization of $f_{i}^{\prime} s$ through $\beta_{i}^{\prime} s$ should be unique. Therefore, $\eta=\xi g$. Thus $\xi:(g, Y) \longrightarrow(\eta, P)$ is a unique morphism in the comma category $(X \downarrow \mathcal{C})$ such that $\beta_{i} \xi=\gamma_{i}:(g, Y) \longrightarrow\left(f_{i}, A_{i}\right)$ for all $i \in I$. Therefore, $\operatorname{GPB}\left[\left(f_{i}, A_{i}\right), \alpha_{i},(f, A)\right]_{I}=\left[(\eta, P), \beta_{i},\left(f, A_{i}\right)\right]_{I}$ in $(X \downarrow \mathcal{C})$. Hence the projection functor $(X \downarrow \mathcal{C}) \longrightarrow \mathcal{C}$.

Dually,

Proposition $1^{*}$. For any category $\mathcal{C}$, the projection functor $(\mathcal{C} \downarrow X) \rightarrow \mathcal{C}$ for the comma category, creates GPO.

Remark 1. If a category $\mathcal{C}$ is a GPB category, then every comma category $(X \downarrow \mathcal{C})$ is also a GPB category.

Remark $1^{*}$. If a category $\mathcal{C}$ is a GPO category, then every comma category $(\mathcal{C} \downarrow X)$ is also a GPO category. 
Proposition 2. The functor $F:$ Morph $\mathcal{C} \rightarrow \mathcal{C} \times \mathcal{C}$ which sends each object $(A, f, B)$ of Morph $\mathcal{C}$ to the object $(A, B)$ in $\mathcal{C} \times \mathcal{C}$, creates GPB as well as GPO.

Proof. The result follows from the fact that all limits are computed pointwise in any functor category [7.5 Schubert].

Indeed, consider $\left[\left(A_{i}, f_{i}, B_{i}\right),\left(\alpha_{i}, \beta_{i}\right),(A, f, B)\right]_{I}$ in Morph $\mathcal{C}$. Then the image of this cone under the functor $F$ is a right cone $\left[\left(A_{i}, B_{i}\right),\left(\alpha_{i}, \beta_{i}\right),(A, B)\right.$ in $\mathcal{C} \times \mathcal{C}$. Suppose its GPB exists in $\mathcal{C} \times \mathcal{C}$ and GPB $\left[\left(A_{i}, B_{i}\right),\left(\alpha_{i}, \beta_{i}\right),(A, B)\right]_{I}=$ $\left[(P, Q),\left(\gamma_{i}, \delta_{i}\right),\left(A_{i}, B_{i}\right)\right]_{I}$. For the left cone $\left[(P, P),\left(\gamma_{i}, f_{i} \gamma_{i}\right),\left(A_{i}, B_{i}\right)\right]_{I}$ in $\mathcal{C} \times \mathcal{C}$, we have

$$
\begin{aligned}
\left(\alpha_{i}, \beta_{i}\right)\left(\gamma_{i}, f_{i} \gamma_{i}\right) & =\left(\alpha_{i} \gamma_{i} \beta_{i}\left(f_{i} \gamma_{i}\right)\right) \\
& =\left(\alpha_{i} \gamma_{i},\left(\beta_{i} f_{i}\right) \gamma_{i}\right) \\
& =\left(\alpha_{i} \gamma_{i},\left(f \alpha_{i}\right) \gamma_{i}\right) \\
& =\left(\alpha_{i} \gamma_{i}, f\left(\alpha_{i} \gamma_{i}\right)\right)
\end{aligned}
$$

for all $i \in I$. Thus $\left(\alpha_{i}, \beta_{i}\right)\left(\gamma_{i}, f_{i} \gamma_{i}\right):(P, P) \rightarrow\left(A_{i}, B_{i}\right) \rightarrow(A, B)$ are the same for all $i \in I$. By $\operatorname{GPB}_{2}$, there exists a unique morphism $\left(I_{P}, g\right)$ : $(P, P) \rightarrow(P, Q)$ in $\mathcal{C} \times \mathcal{C}$ such that $\left(\gamma_{i}, \delta_{i}\right)\left(I_{P}, g\right)=\left(\gamma_{i}, f_{i} \gamma_{i}\right):(P, P) \rightarrow$ $\left(A_{i}, B_{i}\right)$ for all $i \in I$, that is $\delta_{i} g=f_{i} \gamma_{i}$ for all $i \in I$. Now consider, the left cone $\left[(P, g, Q),\left(\gamma_{i}, \delta_{i}\right),\left(A, f_{i}, B_{i}\right)\right]_{I}$ in Morph $\mathcal{C}$. It is clear that $\left(\alpha_{i}, \beta_{i}\right)\left(\gamma_{i}, \delta_{i}\right):(P, g, Q) \rightarrow\left(A_{i}, f_{i}, B_{i}\right) \rightarrow(A, f, B)$ are the same for all $i \in I$ in Morph $\mathcal{C}$. Suppose there is another left cone $\left[(X, h, Y),\left(g_{i}, h_{i}\right),\left(A_{i}, f_{i}, B_{i}\right)\right]$ in Morph $\mathcal{C}$, such that $\left(\alpha_{i}, \beta_{i}\right)\left(g_{i}, h_{i}\right):(X, h, Y) \rightarrow\left(A_{i}, f_{i}, B_{i}\right) \rightarrow(A, f, B)$ are the same for all $i \in I$ in $\mathcal{C} \times \mathcal{C}$. Again by $\mathrm{GPB}_{2}$, there exists a unique morphism $(\eta, \xi):(X, Y) \rightarrow(P, Q)$ in $\mathcal{C} \times \mathcal{C}$ such that $\left(\gamma_{i}, \delta_{i}\right)(\eta, \xi)=\left(\gamma_{i} \eta, \delta_{i} \xi\right)=$ $\left(g_{i}, h_{i}\right)$ for all $i \in I$. For any $i \in I$, we have the diagram

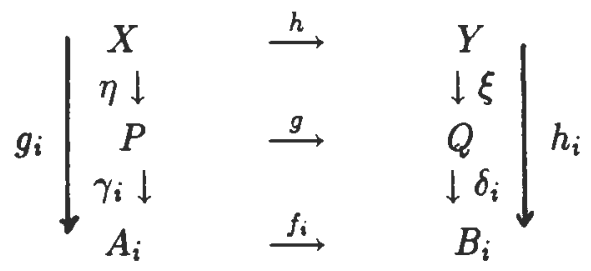

in which outer rectangle and down square are commutative. If we consider the left cone $\left[(X, X),\left(g_{i}, h_{i} h\right),\left(A_{i}, B_{i}\right)\right]_{I}$ in $\mathcal{C} \times \mathcal{C}$, for which $\left(\alpha_{i}, \beta_{i}\right)\left(g_{i}, h_{i} h\right)=$ $\left(\alpha_{i} g_{i},\left(\beta_{i} h_{i}\right) h\right):(X, X) \rightarrow\left(A_{i}, B_{i}\right) \rightarrow(A, B)$ are the same for all $i \in I$. 
Then there are two morphisms $(\eta, \xi h),(\eta, g \eta):(X, X) \rightarrow(P, Q)$ in $\mathcal{C} \times \mathcal{C}$ such that $\left(\gamma_{i}, \delta_{i}\right)(\eta, \xi h)=\left(g_{i}, h_{i} h\right)$ for all $i \in I$ and $\left(\gamma_{i}, \delta_{i}\right)(\eta, g \eta)=\left(g_{i}, h_{i} h\right)$ for all $i \in I$. By uniqueness, we get that $\xi h=g \eta$. Thus $(\eta, \xi):(X, h, Y) \rightarrow$ $(P, g, Q)$ is a morphism in Morph $\mathcal{C}$ such that

$$
\left(\gamma_{i}, \delta_{i}\right)(\eta, \xi)=\left(g_{i}, h_{i}\right):(X, h, Y) \longrightarrow\left(A_{i}, f_{i}, B_{i}\right) \text { for all } i \in I .
$$

This completes the proof that

$$
\operatorname{GPB}\left[\left(A_{i}, f_{i}, B_{i}\right),\left(\alpha_{i}, \beta_{i}\right),(A, f, B)\right]_{I}=\left[(P, g, Q),\left(h_{i}, \delta_{i}\right),\left(A_{i}, f_{i}, B_{i}\right)\right]_{I} .
$$

Hence $F:$ Morph $\mathcal{C} \rightarrow \mathcal{C} \times \mathcal{C}$ creates GPB.

Dually, we can prove that $F$ creates GPO.

Remark 2. If the category $\mathcal{C}$ is a GPB category, then Morph $\mathcal{C}$ is also GPB category and dually.

As a consequence of a result of [3, Theorem $3, p .108]$, we have

Proposition 3. The forgetful functor $U: \mathcal{G} \rightarrow$ Ens creates GPB as well as GPO.

Proposition 4. If $F: \mathcal{A} \rightarrow \mathcal{C}$ is a GPB functor, then for any $X \in \mathcal{C}$ the projection functor $(X \downarrow F) \rightarrow \mathcal{A}$ of the comma category creates GPB.

Proof. Consider a right cone $\left[\left(f_{i}, A_{i}\right), \alpha_{i},(f, A)\right]_{I}$ in the comma category $(X \downarrow F)$, that is

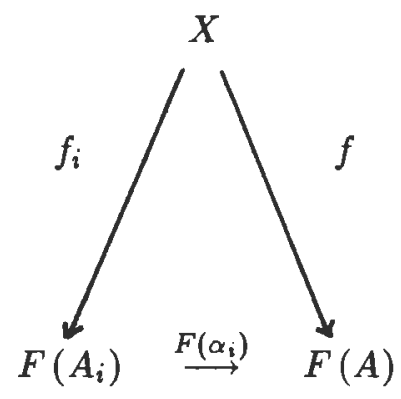

commutes for all $i \in I$. The projection of this cone is a right cone $\left[A_{i}, \alpha_{i}, A\right]_{I}$ in $\mathcal{A}$. 
Suppose, GPB $\left[A_{i}, \alpha_{i}, A\right]_{I}=\left[P, \beta, A_{i}\right]$ in $\mathcal{A}$. Since $F: \mathcal{A} \rightarrow \mathcal{C}$ is a GPB functor, this implies that

$$
\operatorname{GPB}\left[F\left(A_{i}\right), F\left(\alpha_{i}\right), F(A)\right]_{I}=\left[F(P), F\left(\beta_{i}\right), F\left(A_{i}\right)\right]_{I} \text { in } \mathcal{C} .
$$

By commutativity of the above diagram, we get a left cone $\left[X, f_{i}, F\left(A_{i}\right)\right]_{I}$ in $\mathcal{C}$ such that $F\left(\alpha_{i}\right) f_{i}: X \rightarrow F\left(A_{i}\right) \rightarrow F(A)$ are the same for all $i \in I$. By $\mathrm{GPB}_{2}$, there exists a unique morphism $\eta: X \rightarrow F(P)$ in $\mathcal{C}$ such that $F\left(\beta_{i}\right) \eta=f_{i}$ for all $i \in I$. Thus $\left[(\eta, P), \beta_{i},\left(f_{i}, A_{i}\right)\right]_{I}$ is a left cone in $(X \downarrow F)$ such that $\alpha_{i} \beta_{i}:(\eta, P) \rightarrow\left(f_{i}, A_{i}\right) \rightarrow(f, A)$ are the same for all $i \in I$ in $(X \downarrow F)$. Suppose there is a left cone $\left[(g, B), \gamma_{i},\left(f_{i}, A_{i}\right)\right]_{I}$ in $(X \downarrow F)$ such that $\alpha_{i} \gamma_{i}:(g, B) \rightarrow\left(f_{i}, A_{i}\right) \rightarrow(f, A)$ are the same for all $i \in I$. By applying the projection functor, we get that $\alpha_{i} \gamma_{i}: B \rightarrow A_{i} \rightarrow A$ are the same for all $i \in I$ in $\mathcal{A}$. By $\mathrm{GPB}_{2}$, there exists a unique morphism $\phi: B \rightarrow P$ such that $\beta_{i} \phi=\gamma_{i}: B \rightarrow A_{i}$ for all $i \in I$. Now we have to show that $\phi:(g, B) \rightarrow(\eta, P)$ be a morphism in the comma category $(X \downarrow F)$. For any $i \in I$, we have

$$
\begin{array}{rllllll}
X & \stackrel{g}{\longrightarrow} & F(B) & \stackrel{F(\emptyset)}{\longrightarrow} & F(P) & \stackrel{F\left(\beta_{i}\right)}{\longrightarrow} & F\left(A_{i}\right) \\
& =X & \stackrel{g}{\longrightarrow} & F(B) & F\left(\beta_{i} \emptyset\right) & F\left(A_{i}\right) \\
& =X & \stackrel{g}{\longrightarrow} & F(B) & F\left(\gamma_{i}\right) & F\left(A_{i}\right) \\
& =X & \stackrel{f_{i}}{\longrightarrow} & F\left(A_{i}\right) &
\end{array}
$$

By uniqueness of $\eta$, we get $F(\phi) g=\eta$, that is $\phi:(g, B) \rightarrow(\eta, P)$ is a morphism in $(X \downarrow F)$.

Dually,

Proposition 4*. If $F: \mathcal{A} \rightarrow \mathcal{C}$ is a GPO functor, then for any $X \in \mathcal{C}$, the projection functor $(F \downarrow X) \rightarrow \mathcal{A}$ of the comma category creates GPO.

Remark 3. If the category $\mathcal{A}$ is a GPB category, then for any object $X \in \mathcal{C}$, the comma category $(X \downarrow F)$ is also a GPB category and dually.

Remark 4. Proposition 1 and Proposition $1^{*}$ can be considered as corollaries of Proposition 4 and Proposition $4^{*}$ respectively.

Theorem 5. If the functor $F: \mathcal{A} \rightarrow \mathcal{C}$ creates GPB and $\mathcal{C}$ is a GPB category, then $\mathcal{A}$ is also a GPB category and $F$ is GPB functor. 
Proof. By the definition of creation of GPB it is clear that $\mathcal{A}$ is a GPB category, whenever $\mathcal{C}$ is a GPB category and $F$ creates GPB. Let $\left[B_{i}, \beta_{i}, A\right]_{I}$ be an arbitrary right cone and $\operatorname{GPB}\left[B_{i}, \beta_{i}, A\right]_{I 517}=\left\{P, \alpha_{i}, B_{i}\right]_{I}$. Suppose $\operatorname{GPB}\left[F\left(B_{i}\right), F\left(\beta_{i}\right), F(A)\right]_{I}=\left[Q, \gamma_{i}, F\left(B_{i}\right)\right]_{I}$ in $\mathcal{C}$. Since the functor $F$ creates GPB then there exists a unique left cone $\left[P^{\prime}, \gamma_{i}^{\prime}, B_{i}\right]_{I}$ in $\mathcal{A}$ such that $F\left(P^{\prime}\right)=Q, F\left(\gamma_{i}^{\prime}\right)=\gamma_{i}$ for all $i \in I$ and $\left[P^{\prime}, \gamma_{i}^{\prime}, B\right]_{I}=\mathrm{GPB}\left[B_{i}, \beta_{i}, A\right]_{I}$. But GPB is unique up to isomorphism. Then, there exists an isomorphism $\phi: P^{\prime} \cong P$ such that $\alpha_{i} \phi=\gamma_{i}^{\prime}$ for all $i \in I$. This gives $F(\phi): F\left(P^{\prime}\right)=Q \cong$ $F(P)$ is an isomorphism in $\mathcal{C}$ such that $F\left(\alpha_{i}\right) F(\phi)=F\left(\alpha_{i} \phi\right)=F\left(\gamma_{i}^{\prime}\right)=\gamma_{i}$ for all $i \in I$. Thus $\operatorname{GPB}\left[F\left(B_{i}\right), F\left(\beta_{i}\right), F(A)\right]_{I}=\left[F(P), F\left(\alpha_{i}\right), F\left(B_{i}\right)\right]_{I}$. Hence $F$ is a GPB functor.

Dually.

Theorem 5*. If the functor $F: \mathcal{A} \rightarrow \mathcal{C}$ creates GPO and $\mathcal{C}$ is GPO category, then $\mathcal{A}$ is also a GPO category and $F$ is a GPO functor.

Acknowledgement. The authors would like to thank the referee whose valuable comments helped in shaping the into the final form.

\section{References}

[1] M. A. Kazim and S. M. A. Zaidi, "On generalized and super pullbacks", Aligarh Bull. Math. 1, pp. $61-71,(1971)$.

[2] M. A. Kazim and S. M. A. Zaidi, "On characterization of a complete category", Tamkang J. Math. 10, pp. $205-215$, (1979).

[3] S. Maclane, "Categories for the working Mathematician", SpringerVarlag, Graduate Text In Mathematics, (1972). Heldermann Verlag Berlin (1979).

[4] H. Schubert, "Categories", Springer-Verlag, (1972). 
Received : July 03, 1995

H. A. S. Abujabal

M. A. Obaid

Department of Mathematics

Faculty of Science

King Abdul Aziz University

P. O. Box 31464

Jeddah 21497

Saudi Arabia

and

S. M. A. Zaidi

Department of Mathematics

Aligrah Muslim University

Aligrah 202002

India 